\title{
Dental products and services
}

Please send product news information and images to Kate Quinlan at k.quinlan@nature.com,

Product news is provided as a service to readers using text and images from the manufacturer,

supplier or distributor and does not imply endorsement by the BDJ.

Normal and prudent research should be exercised before purchase or use of any product mentioned.

\section{New year, new resolution}

New Year is a time for resolutions, but what if yours is to create even sharper resolution digital images? Dürr Dental's imaging equipment will allow you to do just that and Dürr is so confident in the clarity of its images that they are running a competition throughout 2017 giving away an Apple Watch each month for the best images taken.

Moreover, the best three images from each category, intraoral $\mathrm{X}$-ray, extraoral X-ray and intraoral camera, will also be awarded a brand new VistaCam iX HD package worth up to $£ 4,776$.

Chances are if you're using Dürr equipment you're going to struggle in choosing your best image, which is why the company is allowing each entrant to

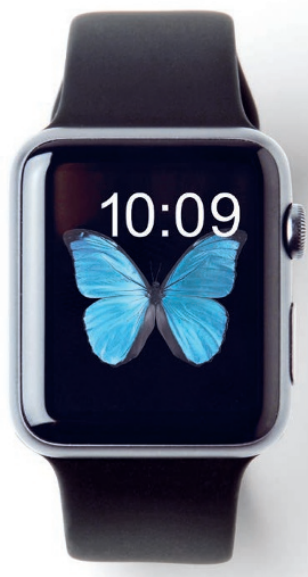
upload up to five images each month. The winners will be chosen by a specialist jury, all of whom have exacting requirements when it comes to quality.

If you haven't experienced Dürr imaging equipment you might like to take a look at some of the uploaded images so you can compare with your own. After all, quality is always relative! For more information or to start uploading images visit www.duerrdental.com/ imagecontest.

\section{No more fuzzy mouths}

Fuzzy Brush has long been known for the chewable toothbrushes it supplies to the hospitality, travel, leisure and airline industries. Now, however, with new product ranges Fuzzy Rock Coolmint, Fuzzy Rock Peppermint and Fuzzy Rock 'Bubble Yum' for kids, Fuzzy Brush has branched out into the retail market.

All of Fuzzy Brush's products contain 95\% xylitol, which promotes dental health by helping to neutralise plaque acidity on teeth and repairing tooth enamel.

Fuzzy Brush's unusual product helps them to reach children and provide a fun way to keep their teeth clean between brushing or for when they're on
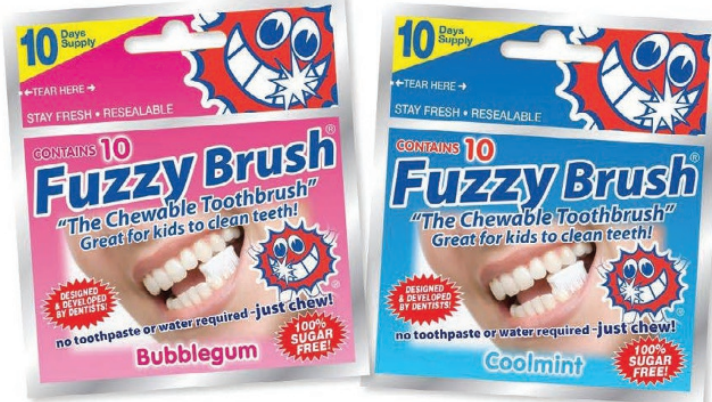
the go. Fuzzy

Brush is clean to promote healthy teeth worldwide and believes in the importance of prevention.

In addition to Fuzzy Brush capsules and resealable packs, Fuzzy Rocks crystal mints are also available in two flavours, bubblegum and coolmint.

Fuzzy Brush supplies more than 1,500 stores in the UK, including Asda, Morrisons, Superdrug, Boots and Lloyds Pharmacists, and are discussing the release of a number of new products in 2017-18 to facilitate demand from others.

www.fuzzybrush.com

\section{Enlightened dentistry}

Innovative UK-based company CALCIVIS has developed a predictable way for dental practitioners to provide true preventive dentistry.

By using a unique and highly specific recombinant, luminescent photoprotein that reacts specifically with free calcium ions - such as those found in actively demineralising tooth surfaces - the CALCIVIS imaging system allows professionals to quickly and effectively at an early stage identify which carious lesions are active and likely to progress.

This precise information can then be used by practitioners to offer a first-response preventive treatment before a cavity can form and more invasive treatments are required.

This technology is unique and is a significant step towards true preventive dentistry - the future of our field. Indeed, protecting a patient's natural teeth is a paramount consideration and professionals must be prepared to focus on the early diagnosis and prompt treatment of dental caries.

With the CALCIVIS imaging system, you can do so.

Expected to launch in the UK in 2017, the CALCIVIS imaging system will change the way dental professionals manage treatment plans for their patients. To find out more, contact the expert team today. Visit www. CALCIVIS.com, call 01316585152 or email info@calcivis.com. 